\title{
Feedback stabilization of discrete-time homogeneous semi-linear systems *
}

\author{
Lars Grüne \\ Fachbereich Mathematik, J.W. Goethe-Universität, Postfach 1119 32, D-60054 Frankfurt a.M., \\ Germany, E-Mail: gruene@math.uni-frankfurt.de \\ Fabian Wirth \\ Zentrum für Technomathematik, Universität Bremen, D-28344 Bremen, Germany, \\ E-Mail: fabian@math.uni-bremen.de
}

For discrete-time semi-linear systems satisfying an accessibility condition asymptotic null controllability is equivalent to exponential feedback stabilizability using a piecewise constant feedback. A constructive procedure that yields such a feedback is presented.

Key words: analytic discrete-time systems, forward accessibility, feedback stabilization, numerical scheme

\section{Introduction}

We consider the problem of feedback stabilizability of homogeneous semi-linear discrete time systems, that is systems linear in the state where the entries of the transition matrix are functions of the control. This class is a generalization of the frequently studied bilinear systems. Systems of this form occur as linearizations with respect to the state only of nonlinear systems at singular points. Also they can be interpreted as systems in which the control affects the parameter of a given system, see also [13].

In recent years there has been considerable progress in this area. For continuous time bilinear systems sufficient conditions for feedback stabilizability have been presented by Ryan and Buckingham [19], Chen et al. [5], Čelikovský [4] and Khapalov and Mohler [15]. In the more general semi-linear case it has been recently shown in Grüne [10] that null controllability is equivalent to feedback stabilizability by discretized feedbacks and a numeric procedure for the calculation of stabilizing feedbacks has been presented. The analysis in this paper

\footnotetext{
* This paper has been written while the first author was a member of the Graduiertenkolleg "Nonlinear Problems in Analysis, Geometry and Physics" (GRK 283) financed by the DFG and the State of Bavaria.
} 
matrices which has been undertaken by Colonius and Kliemann, [7], [8]. Already in [9] the implications on stabilization of this approach have been studied. Furthermore the existence of a classical (measurable) feedback under the assumption of null-controllability is shown by Wang [22]. For general nonlinear systems in continuous time it has been shown in [6] that asymptotic controllability is equivalent to feedback stabilizability by means of a sampled feedback. This approach, however, does not lead to exponential stabilization and is only constructive up to the fact that the knowledge of a control Lyapunov function is required.

Sufficient conditions for feedback stabilizability of bilinear systems in the discrete-time case are presented in Yang et al. [25] and Stepanenko and Yang [21]. The methods employed in these references, however, use in a fundamental way that the system in non-homogeneous, i.e. that the origin is not a common fixed point for all control values. General feedback stabilization schemes for discrete time systems have been presented by Simoes et. al. [3] and Lin and Byrnes [16], [17]. The methods of the latter papers have been used to obtain smooth asymptotically stabilizing feedbacks for bilinear systems in [18] under the assumption that the uncontrolled system is Lyapunov stable.

In this paper we show how the discrete-time version of the results of Colonius and Kliemann which have been presented in Wirth [24] can be used to obtain discrete-time versions of the necessary and sufficient conditions for feedback stabilizability. The proofs are constructive and we discuss numerical aspects of the constructed feedback.

The paper is organized as follows. In Section 2 we present the class of systems we consider and formulate the problem. Furthermore we introduce systems associated to the semi-linear systems, defined on projective space. These systems are vital in the analysis of spectral properties of the original system and for this we review the relevant material from spectral theory of time-varying systems that is needed for the approach in this paper. The main theorem in Section 3 states that feedback stabilizability is characterized by a property of the Floquet spectrum. In the following Section 4 we construct stabilizing feedbacks using methods from optimal control theory to approximate optimal exponential growth rates along trajectories. Section 5 then shows how these results may be used in order to obtain a numerical scheme for the calculation of a piecewise constant exponentially stabilizing feedback. In the final Section 6 we draw conclusions and comment briefly on the robustness properties of the proposed stabilization scheme.

\section{Problem Formulation}

We consider systems on $\mathbb{R}^{d}$ of the form

$$
x(t+1)=A(u(t)) x(t), \quad t \in \mathbb{N},
$$

where $A: \tilde{U} \rightarrow \mathbb{R}^{d \times d}$ is an analytic map, $\tilde{U} \subset \mathbb{R}^{m}$ is open and connected, and the set of admissible control values satisfies $U \subset \tilde{U}$. Let $\Phi(t, u), t \in \mathbb{N}$ denote the evolution operator defined by a sequence $u \in U^{\mathbb{N}}$. We call system (1) asymptotically null-controllable if for every 
to singularity $\Phi(t, u) x=0$ for some finite $t$ may occur. System (1) is called (state) feedback stabilizable if there exists a map $F: \mathbb{R}^{d} \rightarrow U$ such that the system

$$
x(t+1)=A(F(x(t))) x(t), \quad t \in \mathbb{N},
$$

is globally asymptotically stable. If $F$ can be chosen such that (2) is exponentially stable, then we call (1) exponentially (state) feedback stabilizable. It is the purpose of this paper to show that these concepts are equivalent if feedbacks can be chosen to be piecewise constant and to present a procedure for the calculation of exponentially stabilizing feedbacks. Note that it is inherent in this scheme that discontinuous feedbacks may occur.

Before presenting our general approach, let us briefly recall a special case of the results of [18]. In this paper the authors consider systems of the form

$$
x(t+1)=\left(A+\sum_{i=1}^{m} u_{i}(t) B_{i}\right) x(t)+D u(t)=: A x(t)+(B(x(t))+D) u(t)
$$

under the assumption that $A$ is Lyapunov stable, i.e. we may choose $P>0$ such that $A^{T} P A-P \leq 0$. In the following $P$ will always denote a matrix with these properties and furthermore $\|x\|_{P}:=x^{T} P x$. Specializing to $D=0$ we obtain a particular case of $(1)$. In [18] a bounded globally asymptotically stabilizing feedback is shown to exist if for

$$
\begin{gathered}
\Omega:=\left\{x \in \mathbb{R}^{d} \mid\left(A^{s} x\right)^{T}\left(A^{T} P A-P\right) A^{s} x=0, \quad s \geq 0\right\} \\
S:=\left\{x \in \mathbb{R}^{d} \mid\left(A^{s+1} x\right)^{T} P B\left(A^{s} x\right)=0, \quad s \geq 0\right\}
\end{gathered}
$$

we have $\Omega \cap S=\{0\}$. Furthermore an explicit formula for the feedback is given, namely

$$
u(x)=-\left(I+\frac{1}{2} B(x)^{T} P B(x)\right)^{-1} B(x)^{T} P A x
$$

is globally asymptotically stabilizing but clearly in general not exponentially stabilizing. However, exploiting homogeneity $u$ can be modified to be exponentially stabilizing by choosing the feedback to be constant on rays $\left\{\alpha x ; x \in \mathbb{R}^{d} \backslash\{0\}, \alpha>0\right\}$ as can be seen from the following consequence of [18, Theorem 4].

Proposition 1 Consider a homogeneous bilinear system of the form (3) with $D=0$ and assume that $A$ is Lyapunov stable. If $\Omega \cap S=\{0\}$ then for any $\rho>0$ the system (3) is exponentially stabilized by the feedback defined by $F_{\rho}(0)=0$ and

$$
F_{\rho}(x)=-\left[I+\frac{1}{2} B\left(\frac{\rho x}{\|x\|_{P}}\right)^{T} P B\left(\frac{\rho x}{\|x\|_{P}}\right)\right]^{-1} B\left(\frac{\rho x}{\|x\|_{P}}\right)^{T} P A \frac{\rho x}{\|x\|_{P}} .
$$

Note that for every $\rho>0 F_{\rho}$ is smooth on $\mathbb{R}^{d} \backslash\{0\}$. Furthermore $F_{\rho}$ is bounded and the bound can be made arbitrarily small by choosing $\rho$ small. 
feedbacks $u$ in $(4)$ and $F_{\rho}$ by $x(\cdot ; u)$ resp. $x\left(\cdot ; F_{\rho}\right)$. It is shown in $[18$, Theorem 4$]$ that

$$
\|x(t+1 ; u)\|_{P}^{2}-\|x(t ; u)\|_{P}^{2} \leq-2\|u(x(t))\|^{2}
$$

holds. By the assumption $\Omega \cap S=\{0\}$ it may be shown that for $x(t ; u) \neq 0$ there exists $s \geq 0$ such that either $u(x(\tau ; u)) \neq 0$ for some $\tau \in\{t, \ldots, t+s+1\}$ or $\|x(t+s+1 ; u)\|_{P}<\|x(t ; u)\|_{P}$. Using continuity and compactness it follows that there exists a $T \geq 0$ and a constant $1>\beta>0$ such that for any $\|x(0 ; u)\|_{P}=\rho$ we have $\|x(T ; u)\|_{P}^{2}-\|x(0 ; u)\|_{P}^{2}<-\beta\|x(0 ; u)\|_{P}^{2}$. By the homogeneity of the closed loop system defined by $F_{\rho}$ and the fact that $F_{\rho}(x)=u(x)$ for $\|x\|_{P}=\rho$ this implies

$$
\left\|x\left(t+T ; F_{\rho}\right)\right\|_{P}^{2}-\left\|x\left(t ; F_{\rho}\right)\right\|_{P}^{2} \leq-\frac{\beta}{\rho}\left\|x\left(t ; F_{\rho}\right)\right\|_{P}^{2}
$$

Hence exponential stabilization by the feedback $F_{\rho}$ follows.

It is a further interesting fact that for the feedbacks $F_{\rho}$ just defined we have $F_{\rho}(x)=F_{\rho}(-x)$. Thus $F_{\rho}$ does define a smooth map on the projective space $\mathbb{P}^{d-1}$, and in fact our general approach uses feedback maps induced by maps on $\mathbb{P}^{d-1}$. As we will see these kind of maps suffice for the feedback stabilization of (1). The basic idea of the construction of the feedback is to obtain upper bounds on the exponential growth rates of the trajectories using ideas from optimal control. In a more general situation than the one considered in Proposition 1 however one cannot expect to obtain smooth or even continuous feedbacks using this approach. Also our approach does in general not yield explicit formulas.

We equip $\mathbb{P}^{d-1}$ with a Riemannian metric $d(\cdot, \cdot)$. Let $\mathbb{P}$ denote the natural projection of a subset in $\mathbb{R}^{d} \backslash\{0\}$ to $\mathbb{P}^{d-1}$. A matrix $A \in \mathbb{R}^{d \times d}$ defines a map $\mathbb{P} A: \mathbb{P}^{d-1} \backslash \mathbb{P} \operatorname{ker} A \rightarrow \mathbb{P} \operatorname{Im} A$ as $A$ maps one-dimensional subspaces into one-dimensional subspaces (or to 0 ). In homogeneous coordinates this means $\eta=\mathbb{P} A \xi$ iff $\xi=[x], A x \neq 0, \eta=[A x]$, where we have taken the usual equivalence relation on $\mathbb{R}^{d} \backslash\{0\}$ given by $x \sim y$ iff $\exists \gamma \neq 0: x=\gamma y$ and $[x]$ denotes the equivalence class of $x$. With these remarks the associated system to (1) is given by

$$
\begin{aligned}
\xi(t+1) & =\mathbb{P} A(u(t)) \xi(t), \quad t \in \mathbb{N} \\
\xi(0) & =\xi_{0} \in \mathbb{P}^{d-1}, \quad u \in U^{\mathbb{N}}\left(\xi_{0}\right) .
\end{aligned}
$$

Here $U^{\mathbb{N}}\left(\xi_{0}\right)$ denotes the set of admissible control sequences for $\xi \in \mathbb{P}^{d-1}$, i.e. those control sequences $u \in U^{\mathbb{N}}$ such that $\Phi(t, u) x_{0} \neq 0$ for all $t \in \mathbb{N}$, whenever $\mathbb{P} x_{0}=\xi_{0}$. The solution of (6) corresponding to an initial value $\xi$ and a control sequence $u$ is denoted by $\varphi(\cdot ; \xi, u)$. In order to be able to use the results obtained in [24] we assume that the map $A$ and the sets $U \subset \tilde{U} \subset \mathbb{R}^{m}$ satisfy:

(A) The set $U_{\text {inv }}:=\{u \in U \mid \operatorname{det}(A(u)) \neq 0\}$ is not empty.

(B) $U$ is compact with connected interior.

(C) $U \subset \operatorname{clint} U \subset \tilde{U}$. 
definition of the forward orbit of a point $\xi$ given by

$$
\mathcal{O}^{+}(\xi):=\left\{\eta \in \mathbb{P}^{d-1} \mid \exists t \in \mathbb{N}, u \in U^{t} \text { such that } \eta=\varphi(t ; \xi, u)\right\}
$$

The following concept introduces regions of approximate controllability in $\mathbb{P}^{d-1}$. A control set is a set $D \subset \mathbb{P}^{d-1}$ satisfying

(i) $D \subset \operatorname{clO}^{+}(\xi)$ for all $\xi \in \mathbb{P}^{d-1}$.

(ii) int $D \neq \emptyset$.

(iii) $D$ is a maximal set (with respect to inclusion) satisfying (i).

It is also possible to consider control sets with empty interior, but for the purposes of this paper this is unnecessary. Control sets have been studied in [1] and [24] and the references therein. We now present some of the relevant facts.

If we assume that $(6)$ is forward accessible, i.e. int $\mathcal{O}^{+}(\xi) \neq \emptyset$ for every $\xi \in \mathbb{P}^{d-1}$, then there exists a unique invariant control set in $\mathbb{P}^{d-1}$, i.e. a unique set $C$ satisfying $\operatorname{cl} C=\mathrm{cl}^{+}(\xi)$, $\forall \xi \in C . C$ is closed, connected and has nonempty interior. An important subset of a control set $D$ is its core defined by

$$
\operatorname{core}(D):=\left\{\xi \in D \mid \operatorname{int} \hat{\mathcal{O}}^{-}(\xi) \cap D \neq \emptyset \text { and } \operatorname{int} \hat{\mathcal{O}}^{+}(\xi) \cap D \neq \emptyset\right\}
$$

Here $\hat{\mathcal{O}}^{-}(\xi)$ denotes the points $\eta \in \mathbb{P}^{d-1}$ for which there exist $t \in \mathbb{N}, u_{0} \in \operatorname{int} U^{t}$ such that $\varphi\left(t ; \eta, u_{0}\right)=\xi$ and the map $u \mapsto \varphi(t ; \eta, u)$ has full rank in $u_{0}$. Under these conditions $\left(\eta, u_{0}\right)$ is called a regular pair. A control $u \in \operatorname{int} U^{t}$ is called universally regular if $(\xi, u)$ is a regular pair for all $\xi \in \mathbb{P}^{d-1}$. By [20, Corollaries $\left.3.2 \& 3.3\right]$ forward accessibility is equivalent to the fact that the set of universally regular control sequences is open and dense in $U^{t}$ for all $t$ large enough. $\hat{\mathcal{O}}^{+}(\xi)$ is defined by $\eta \in \hat{\mathcal{O}}^{+}(\xi)$ iff $\xi \in \hat{\mathcal{O}}^{-}(\eta)$.

To the invariant control set $C$ we may associate a set of Floquet exponents by

$$
\Sigma_{F l}(C):=\left\{\frac{1}{t} \log |\lambda| \mid \lambda \in \sigma(\Phi(t, u)), u \in U^{t}, \mathbb{P} G E(\lambda, u) \subset \operatorname{core}(C)\right\} .
$$

where we use the convention $\log 0=-\infty$. Here $\sigma(\Phi(t, u))$ denotes the spectrum of $\Phi(t, u)$ and $G E(\lambda, u)$ denotes the generalized eigenspace of an eigenvalue $\lambda \in \sigma(\Phi(t, u))$ or in the case of complex $\lambda$ the kernel of the (real) matrix $((\lambda I-\Phi(t, u))(\bar{\lambda} I-\Phi(t, u)))^{d}$.

The meaning of the Floquet exponents becomes clear if we introduce the exponential growth rate of a trajectory which is measured by the Lyapunov exponent

$$
\lambda\left(x_{0}, u\right):=\limsup _{t \rightarrow \infty} \frac{1}{t} \log \left\|\Phi(t, u) x_{0}\right\| .
$$

Clearly, $\lambda\left(x_{0}, u\right)<0$ iff the corresponding trajectory converges to 0 exponentially fast, as $\lambda$ measures the exponential growth of a trajectory. Due to the linearity (in $x$ ) of system (1) 


\section{Main result}

It is an easy consequence of [24, Theorem 11.1] that the infimum of $\Sigma_{F l}(C)$ characterizes exponential null controllability as it may be seen that

$$
\sup _{\xi \in \mathbb{P}^{d-1}} \inf _{u \in U^{\mathbb{N}}} \lambda(\xi, u)=\inf \Sigma_{F l}(C)
$$

where possibly both sides are equal to $-\infty$. In fact, the infimum of the Floquet spectrum over $C$ also characterizes asymptotic null controllability and feedback stabilizability as the following main theorem states.

Theorem 2 Let (A),(B),(C) hold and assume that (6) is forward accessible, then the following statements are equivalent.

(i) System (1) is asymptotically null controllable.

(ii) System (1) is feedback stabilizable with a piecewise constant feedback F.

(iii) System (1) is exponentially feedback stabilizable with a piecewise constant feedback $F$.

(iv) $\inf \Sigma_{F l}(C)<0$.

PROOF. (i) $\Rightarrow$ (iv): Pick a point $\xi_{0} \in \operatorname{core}(C)$. By [24, Lemma 10.1] there exists a time $T$ such that for every point $\xi \in C$ there exists a control sequence $u_{\xi} \in U^{T}$ with $\varphi\left(t_{\xi}, \xi, u_{\xi}\right)=\xi_{0}$ for some $t_{\xi} \leq T$. By the boundedness of $\|A(u)\|, u \in U$ from above we can conclude that for all $x \in \mathbb{R}^{d}$ with $\mathbb{P} x=\xi$ the estimate $\left\|\Phi\left(t_{\xi}, u_{\xi}\right) x\right\| \leq K\|x\|$ is valid for some constant $K>0$ independent of $x$. Also, asymptotic null controllability implies that there exists a time $t_{0}>0$ and a control function $u_{0} \in U^{t_{0}}$ such that

$$
\left\|\Phi\left(t_{0}, u_{0}\right) x_{0}\right\| \leq \frac{1}{2 K}\left\|x_{0}\right\|
$$

for all $x_{0} \in \mathbb{R}^{d}$ with $\mathbb{P} x_{0}=\xi_{0}$. Now denote $x_{1}:=\Phi\left(t_{0}, u_{0}\right) x_{0}$. If $x_{1}=0$ it follows that $\inf \Sigma_{F l}(C)=-\infty$ and we are done. Otherwise by invariance of $C \xi_{1}:=\mathbb{P} x_{1} \in C$. Choose $u_{1} \in U^{t_{1}}$ steering to $\xi_{0}$ in time $t_{1} \leq T$. Concatenating $u_{0}$ and $u_{1}$ we obtain a control $u \in U^{t_{0}+t_{1}}$ satisfying

$$
\varphi\left(t_{0}+t_{1}, \xi_{0}, u\right)=\xi_{0} \quad \text { and } \quad\left\|\Phi\left(t_{0}+t_{1}, u\right) x_{0}\right\| \leq \frac{1}{2}\left\|x_{0}\right\|
$$

This implies $-\frac{1}{\left(t_{0}+t_{1}\right)} \log 2 \in \mathrm{cl} \Sigma_{F l}(C)$ and the assertion follows. (iv) $\Rightarrow$ (iii) follows from Theorem 11 below, while (iii) $\Rightarrow$ (ii) and (ii) $\Rightarrow$ (i) are immediately clear. 
a numerical point of view. We circumvent these problems by introducing approximations of (1) by invertible systems. We consider sequences $\left\{U_{n}\right\}_{n \in \mathbb{N}}$ of compact sets satisfying for each $n \in \mathbb{N}$ the following condition:

$$
\operatorname{int} U_{n} \neq \emptyset, \quad \operatorname{cl} U_{n} \subset \operatorname{int}_{U} U_{n+1} \subset U_{i n v}, \quad \bigcup_{n \in \mathbb{N}} U_{n}=U_{i n v},
$$

where int $U$ denotes the interior in the relative topology of $U$. One possible choice of such a sequence $\left\{U_{n}\right\}$ can be obtained via the following procedure. Let $B(\varepsilon):=\{u \in U \mid \operatorname{det}(A(u)) \geq$ $\varepsilon\}$. Then we may choose $\varepsilon>0$ such that $\operatorname{int} B(\varepsilon) \neq \emptyset$. Now define $U_{n}:=B(\varepsilon / n)$ for $n \geq 1$.

Assuming (8) we consider the approximating systems

$$
\begin{array}{r}
\xi(t+1)=\mathbb{P} A(u(t)) \xi(t) \\
\xi_{0} \in \mathbb{P}^{d-1}, \quad u \in U_{n}^{\mathbb{N}} .
\end{array}
$$

The following proposition states in what sense the systems $\left(6_{n}\right)$ are appropriate approximations of the original system.

Proposition 3 Let $\left\{U_{n}\right\}_{n \in \mathbb{N}}$ be a family of sets satisfying (8) then

(i) For every $n \in \mathbb{N}$ system $\left(\sigma_{n}\right)$ is forward accessible.

(ii) For every $n \in \mathbb{N}$ system $\left(6_{n}\right)$ has a unique invariant control set $C_{n}$.

(iii) $\lim _{n \rightarrow \infty} \inf \Sigma_{F l}\left(C_{n}\right)=\inf _{n \in \mathbb{N}} \inf \Sigma_{F l}\left(C_{n}\right)=\inf \Sigma_{F l}(C)$.

(iv) System (6) is asymptotically null-controllable iff there exists an $n_{0} \in \mathbb{N}$ such that system $\left(6_{n}\right)$ is asymptotically null-controllable for all $n \geq n_{0}$.

PROOF. (i) By [20, Corollaries $3.2 \& 3.3]$ forward accessibility of (6) and int $U_{n} \neq \emptyset$ imply that for $t$ large enough (independently of $n$ ) there is a universally regular control sequence $u_{n} \in \operatorname{int} U_{n}^{t}$. This implies that $\varphi\left(t ; \xi, u_{n}\right) \in \operatorname{int} \mathcal{O}^{+}(\xi)$ for all $\xi \in \mathbb{P}^{d-1}$, which shows forward accessibility.

(ii) Using the control $u_{n}$ from part (i), let $V\left(u_{n}\right)$ denote the sum of the eigenspaces of $\Phi\left(t, u_{n}\right)$ corresponding to the eigenvalues of greatest modulus, i.e.

$$
V\left(u_{n}\right):=\bigoplus_{\lambda \in \sigma\left(\Phi\left(t, u_{n}\right)\right),|\lambda|=r\left(\Phi\left(t, u_{n}\right)\right)} G E\left(\lambda, u_{n}\right) .
$$

Correspondingly, define

$$
W\left(u_{n}\right):=\bigoplus_{\lambda \in \sigma\left(\Phi\left(t, u_{n}\right)\right),|\lambda|<r\left(\Phi\left(t, u_{n}\right)\right)} G E\left(\lambda, u_{n}\right)
$$

By [24, Proposition 6.7] there is a control set $C_{n}$ such that $\mathbb{P} V\left(u_{n}\right) \subset \operatorname{core}\left(C_{n}\right)$. We claim that $C_{n}$ is the unique invariant control set of $\left(6_{n}\right)$. Note that for $\xi \in \mathbb{P}^{d-1} \backslash W\left(u_{n}\right)$ it holds 


$$
\lim _{k \rightarrow \infty} d\left(\mathbb{P} \Phi\left(t, u_{n}\right)^{k} \xi, V\left(u_{n}\right)\right)=0
$$

As system $\left(6_{n}\right)$ is forward accessible and int $\mathbb{P} W\left(u_{n}\right)=\emptyset$ this shows that

$$
\mathbb{P} V\left(u_{n}\right) \cap \operatorname{cl}^{+}(\xi) \neq \emptyset \text { for all } \xi \in \mathbb{P}^{d-1} .
$$

One the one hand this shows that $C_{n}$ is invariant, as any trajectory with initial condition $\xi_{0} \in C_{n}$ can be steered back to core $\left(C_{n}\right)$ such that the whole trajectory is contained in $C_{n}$ by maximality of control sets. On the other hand there is no other invariant control set $C^{\prime}$ as (9) shows that from some control set $C^{\prime} \neq C_{n}$ it is possible to steer to $C_{n}$ contradicting the invariance of $C^{\prime}$.

(iii) As $U_{n} \subset U_{n+1} \subset U$ it follows that $C_{n} \subset C_{n+1} \subset C$ and $\inf \Sigma_{F l}\left(C_{n}\right) \geq \inf \Sigma_{F l}\left(C_{n+1}\right) \geq$ inf $\Sigma_{F l}(C)$. Thus it follows that $\lim _{n \rightarrow \infty} \inf \Sigma_{F l}\left(C_{n}\right)$ exists and

$$
\lim _{n \rightarrow \infty} \inf \Sigma_{F l}\left(C_{n}\right)=\inf _{n \in \mathbb{N}} \inf \Sigma_{F l}\left(C_{n}\right) \geq \inf \Sigma_{F l}(C)
$$

To prove the remaining inequality fix $c>\inf \Sigma_{F l}(C)$. Choose $t \in \mathbb{N}$, and a universally regular $u \in U_{\text {inv }}^{t}$ such that there exists $\lambda \in \sigma(\Phi(t, u))$ with $\mathbb{P} G E(\lambda, u) \subset \operatorname{core}(C)$ and $\frac{1}{t} \log |\lambda|<c$. By universal regularity and the proof of (ii) we have $\mathbb{P} V(u) \subset \operatorname{core}(C)$. For $\xi \in \mathbb{P} V(u)$ and $\eta \in \mathbb{P} G E(\lambda, u)$ we may by $\left[24\right.$, Lemma 10.1 (ii)] choose a universally regular control $v \in U_{\text {inv }}^{s}$ such that

$$
\eta=\varphi(s ; \xi, v)
$$

As $u \in U_{i n v}^{t}, v \in U_{i n v}^{s}$ there exists an $n_{0} \in \mathbb{N}$ such that $u \in \operatorname{int} U_{n}^{t}, v \in \operatorname{int} U_{n}^{s}$ holds for all $n \geq n_{0}$. By part (ii) we have $V(u) \subset \operatorname{core}\left(C_{n}\right)$ and as the control $v$ is available for $\left(6_{n}\right)$ the invariance of $C_{n}$ implies that $\eta \in C_{n}$. On the other hand again using [24, Proposition 6.7] there is a control set $D$ such that $\mathbb{P} G E(\lambda, u) \subset D$. Now $D \cap C_{n} \neq \emptyset$ and hence $D=C_{n}$ by the maximality of control sets. By definition of the Floquet spectrum if follows that $\frac{1}{t} \log |\lambda| \in \Sigma_{F l}\left(C_{n}\right)$ and so inf $\Sigma_{F l}\left(C_{n}\right)<c$. As $c>\inf \Sigma_{F l}(C)$ was arbitrary this completes the proof.

(iv) Clearly if $\left(6_{n_{0}}\right)$ is asymptotically null-controllable then the same holds for all $n \geq$ $n_{0}$ and (6). It has already been shown in the implication (i) $\Rightarrow$ (iv) of Theorem 2 that asymptotic null-controllability of $(6)$ implies that $\inf \Sigma_{F l}(C)<0$. Hence for all $n$ large enough inf $\Sigma_{F l}\left(C_{n}\right)<0$. Now (7) shows the claim. Note that the proof did not depend on the connectedness of $U$.

Finally, we have to point out in this section that inf $\Sigma_{F l}(C)<0$ does not imply that there exists a periodic sequence $u$ such that the spectral radius satisfies $r(\Phi(t, u))<1$. So that constructing a stabilizing feedback is not equivalent to the possibility of choosing a stable periodic system in the family (1). 


$$
A(a, b)=\left[\begin{array}{cr}
a & 1 \\
a b-1 & b
\end{array}\right]
$$

Let $U=[-2,2]^{2}$. Clearly, for all $u=(a, b) \in U$ it holds that $\operatorname{det}(A(u))=1$, hence $\operatorname{det}(\Phi(t, u))=1$ and thus $r(\Phi(t, u)) \geq 1$ for all $t \in \mathbb{N}, u \in U^{t}$. Thus a periodic system in the family (1) is never exponentially stable.

However, for every $T \in \operatorname{sl}(2, \mathbb{R})$, the group of real $2 \times 2$ matrices with determinant 1 , there exist $t \in \mathbb{N}$ and $u \in U^{t}$ such that $T=\Phi(t, u)$. This can be shown by a simple calculation.

This means that in this case the projection (6) is completely controllable on $\mathbb{P}^{1}$. Thus $C=\mathbb{P}^{1}$ and $\inf \Sigma_{F l}(C) \leq-\log 2$ as $\sigma(A(2,1 / 2))=\{2,1 / 2\}$.

Note also that the question, whether there exists a stable periodic system in the family (1) is in general algorithmically undecidable, as has been shown in [2], whereas the problem of approximating inf $\Sigma_{F l}(C)$ has an algorithmic solution as will be shown in the remainder of this article.

\section{Construction of the Feedback}

In this section we will give a constructive approach for the calculation of the exponentially stabilizing feedback for system (1). It is based on a dynamic programming approach, using the fact that optimal exponential growth rates can be approximated by discounted values along trajectories.

The construction of the feedback is related to the following optimal control problem: Define the function $q: \mathbb{P}^{d-1} \times U \rightarrow \mathbb{R} \cup\{-\infty\}$ by

$$
q(\xi, u):=\left\{\begin{array}{cl}
\log \frac{\|A(u) x\|}{\|x\|}, & u \in U(\xi), \xi=\mathbb{P} x \\
-\infty, & \text { else }
\end{array}\right.
$$

and the running cost $J_{0}: \mathbb{P}^{d-1} \times U^{\mathbb{N}} \rightarrow \mathbb{R} \cup\{-\infty\}$ given by

$$
J_{0}(\xi, u):=\left\{\begin{array}{cl}
\limsup _{t \rightarrow \infty} \frac{1}{t} \sum_{s=0}^{t-1} q(\varphi(s ; \xi, u), u(s)), & u \in U^{\mathbb{N}}(\xi), \\
-\infty & \text { else. }
\end{array}\right.
$$

Note that $q$ and thus also the sum over $q$ are bounded from above. 
$v_{0}(\xi):=\inf \left\{J_{0}(\xi, u) \mid u \in U^{\mathbb{N}}\right\}$ is negative for all points in the projective space. $v_{0}$ may be approximated by value functions $v_{\delta}$ corresponding to the following $\delta$-discounted yield:

$$
J_{\delta}(\xi, u):=\left\{\begin{array}{cl}
\limsup _{t \rightarrow \infty} \sum_{s=0}^{\infty} \mathrm{e}^{-\delta t} q(\varphi(s ; \xi, u), u(s)), u \in U^{\mathrm{N}}(\xi), \\
-\infty & \text { else. }
\end{array}\right.
$$

We will also consider the value functions corresponding to the approximations $U_{n}$, given by

$$
v_{\delta, n}(\xi):=\inf _{u \in U_{n}^{\mathbb{N}}} J_{\delta}(\xi, u), \quad v_{0, n}(\xi):=\inf _{u \in U_{n}^{\mathbb{N}}} J_{0}(\xi, u)
$$

Note that the series in the definition of $J_{\delta}$ is divergent iff the partial sums tend to $-\infty$, and that by assumption (8) we have $\inf _{\xi \in \mathbb{P}^{d-1}} v_{\delta, n}(\xi)>-\infty$.

Theorem 5 Consider system (1) and assume that its associated system (6) is forward accessible, then it holds that

$$
\lim _{\delta \rightarrow 0} \max _{\xi \in \mathbb{P}^{d-1}}\left(1-e^{-\delta}\right) v_{\delta}(\xi)=\max _{\xi \in \mathbb{P}^{d-1}} v_{0}(\xi)=\inf \Sigma_{F l}(C)
$$

Furthermore, it holds for all $n \in \mathbb{N}$, that

$$
\lim _{\delta \rightarrow 0} \max _{\xi \in \mathbb{P}^{d-1}}\left(1-e^{-\delta}\right) v_{\delta, n}(\xi)=\max _{\xi \in \mathbb{P}^{d-1}} v_{0, n}(\xi)=\inf \Sigma_{F l}\left(C_{n}\right)
$$

PROOF. The equalities on the right hand side in each statement follow from (7).

We now obtain an upper bound for $\lim \sup _{\delta \rightarrow 0} \max _{\xi \in \mathbb{P}^{d-1}}\left(1-e^{-\delta}\right) v_{\delta, n}(\xi)$. The same argument can be applied to $v_{\delta}$ and this case is therefore omitted. Fix $n \in \mathbb{N}$ and choose $\xi_{0} \in \operatorname{core}\left(C_{n}\right)$. By [24, Lemma 10.1 (ii)] there exists a time $T_{0} \in \mathbb{N}$ such that every point $\xi \in \mathbb{P}^{d-1}$ can be controlled to $\xi_{0}$ in a time $t=t(\xi) \leq T_{0}$ by some control $u=u(\xi)$. Using this $u$ it follows from Bellman's principle of optimality that

$$
v_{\delta, n}(\xi) \leq \sum_{s=0}^{t-1} \mathrm{e}^{-\delta s} q(\varphi(s ; \xi, u), u(s))+\mathrm{e}^{-\delta t} v_{\delta, n}\left(\xi_{0}\right)
$$

and hence there exists $\varepsilon(\delta)$ such that for all $\xi \in \mathbb{P}^{d-1}$ we have $\left(1-e^{-\delta}\right) v_{\delta, n}(\xi) \leq(1-$ $\left.e^{-\delta}\right) v_{\delta, n}\left(\xi_{0}\right)+\varepsilon(\delta)$ where $\varepsilon(\delta) \rightarrow 0$ as $\delta \rightarrow 0$. By [23, Corollary 3.5] it holds that (1$\left.e^{-\delta}\right) v_{\delta, n}\left(\xi_{0}\right) \leq v_{0, n}\left(\xi_{0}\right)+\tilde{\varepsilon}(\delta)$ where again $\tilde{\varepsilon}(\delta) \rightarrow 0$ as $\delta \rightarrow 0$. Together this implies

$$
\limsup _{\delta \rightarrow 0} \max _{\xi \in \mathbb{P}^{d-1}}\left(1-e^{-\delta}\right) v_{\delta, n}(\xi) \leq \max _{\xi \in \mathbb{P}^{d-1}} v_{0, n}(\xi) .
$$

The proof of the first statement now follows from [23, Theorem 4.9], where convergence of $\delta v_{\delta}$ to $v_{0}$ on core $(C)$ is shown. Now assume $\lim \inf _{\delta \rightarrow 0} \max _{\xi \in \mathbb{P}^{d-1}} \delta v_{\delta, n}(\xi)=\sigma<\max _{\xi \in \mathbb{P}^{d-1}} v_{0, n}(\xi)$, then [23, Proposition 3.8] and the boundedness of $q$ on $\mathbb{P}^{d-1} \times U_{n}$ yield a contradiction. 
using optimal feedbacks for the discounted problem. We will now show that this is indeed the case. We construct a feedback as follows.

Definition 6 Define $F_{\delta, n}: \mathbb{P}^{d-1} \rightarrow U_{n}$ by the following procedure:

For each $\xi \in \mathbb{P}^{d-1}$ choose a value $u \in U_{n}$ such that

$$
q(\xi, u)+\mathrm{e}^{-\delta} v_{\delta, n}(\varphi(1 ; \xi, u))
$$

becomes minimal and let $F_{\delta, n}(\xi):=u$.

The function $F_{\delta, n}$ will in general not be unique; nevertheless the existence of a value $F_{\delta, n}(\xi)$ with the desired properties is always guaranteed by the continuity of $q, v_{\delta, n}$ and $u \mapsto \varphi(1 ; \xi, u)$ and the compactness of $U_{n}$. Denote the solution of the system using $F_{\delta, n}$ by $\varphi\left(\cdot ; \xi, F_{\delta, n}\right)$. It is a straightforward calculation to show that this feedback law is indeed an optimal control strategy for $v_{\delta, n}$, i.e. it holds that

$$
J_{\delta}\left(\xi_{0}, F_{\delta, n}\right):=\sum_{s=0}^{\infty} \mathrm{e}^{-\delta s} q\left(\varphi\left(s ; \xi_{0}, F_{\delta, n}\right), F\left(\varphi\left(s ; \xi_{0}, F_{\delta, n}\right)\right)\right)=v_{\delta, n}\left(\xi_{0}\right)
$$

It turns out, however, that this feedback is also exponentially stabilizing.

Theorem 7 Assume that (1) is asymptotically null-controllable and (6) is forward accessible, then there exists an $n_{0} \in \mathbb{N}$ and $a \delta_{0}>0$ such that for all $n \geq n_{0}$ and $0<\delta \leq \delta_{0}$ the feedback $F: \mathbb{R}^{d} \rightarrow U_{n}$ given by $F(0)=u_{0}$ for some arbitrary $u_{0} \in U_{n}$ and

$$
F(x)=F_{\delta, n}(\xi) \quad \text { iff } \quad \mathbb{P} x=\xi
$$

exponentially stabilizes system (1).

PROOF. Let $n_{0}$ be such that $\inf \Sigma_{F l}\left(C_{n_{0}}\right)<0$ and choose $\delta_{0}$ such that $\max _{\xi \in \mathbb{P}^{d-1}} v_{\delta, n_{0}}(\xi)<$ 0 for all $0<\delta<\delta_{0}$. Then $\inf \Sigma_{F l}\left(C_{n}\right)<0$ and $\max _{\xi \in \mathbb{P}^{d-1}} v_{\delta, n}(\xi)<0$ for all $(n, \delta)$ with $n \geq n_{0}$ and $0<\delta \leq \delta_{0}$. Choose $n \geq n_{0}, 0<\delta \leq \delta_{0}$. Denote the exponential growth rate of an initial condition $x_{0}$ under the feedback $F$ by $\lambda\left(x_{0}, F\right)$. For any initial condition $x_{0} \neq 0$ we have

$$
\begin{gathered}
\lambda\left(x_{0}, F\right)=\lambda\left(\mathbb{P} x_{0}, F_{\delta, n}\right) \leq \limsup _{t \rightarrow \infty}\left(1-\mathrm{e}^{-\delta}\right) J_{\delta}\left(\varphi\left(t ; \mathbb{P} x_{0}, F_{\delta, n}\right), F_{\delta, n}\right) \\
\leq \max _{\xi \in \mathbb{P}^{d-1}}\left(1-\mathrm{e}^{-\delta}\right) v_{\delta, n}(\xi)<0,
\end{gathered}
$$

where we used [23, Proposition 3.8] and the fact that $q$ is bounded on $\mathbb{P}^{d-1} \times U_{n}$. To complete the proof it suffices to show the existence of a constant $M>1$ such that $\left\|x\left(t ; x_{0}, F\right)\right\| \leq M e^{\beta t}$, 
from (10) the existence of a $T$ such that

$$
\frac{1}{t(\xi)} \sum_{s=0}^{t(\xi)} q\left(\varphi\left(s+t ; \xi, \tilde{F}_{\alpha}\right), \tilde{F}_{\alpha}\left(\varphi\left(s+t ; \xi, \tilde{F}_{\alpha}\right)\right)\right) \leq \beta+\varepsilon
$$

for all $\xi \in \mathbb{P}^{d-1}$ and some $t(\xi) \leq T$. By induction and boundedness of $A(U)$ exponential stability follows.

\section{A Numerical Construction of the Feedback}

Usually, it will not be possible to calculate $v_{\delta, n}$ explicitly. Instead we assume that we are given a numerical approximation to this optimal value function. From now on assume we have fixed a compact control range $U_{n} \subset U_{\text {inv }}$ which approximates our original control problem to a desired accuracy. The main implication of this is the existence of a constant $M_{q}$ such that $|q(\xi, u)| \leq M_{q}$ for all $\xi \in \mathbb{P}$ and all $u \in U_{n}$.

A numerical approximation of $v_{\delta, n}$ can be obtained as in [11]: Parameterizing $\mathbb{P}^{d-1}$ in a suitable way we obtain a transformation of the problem to some subset $\Omega \subset \mathbb{R}^{d-1}$ on which we have to solve a discrete Hamilton-Jacobi-Bellman equation. The solution of this equation can be approximated on a grid covering $\Omega$ where we look for a solution which is piecewise linear on each element of the grid. This solution can be calculated iteratively and, using the inverse of the parameterization, gives an approximation of $v_{\delta, n}$ on $\mathbb{P}^{d-1}$. We denote this piecewise linear numerical approximation by $\tilde{v}_{\delta}$. Using the results from [12] $\tilde{v}_{\delta}$ may be calculated in such a way that

$$
\tilde{v}_{\delta}(\xi)=\inf _{u \in U_{n}}\left\{q(\xi, u)+\mathrm{e}^{-\delta} \tilde{v}_{\delta}(\varphi(1 ; \xi, u))\right\}+\eta(\xi)
$$

where $|\eta(\xi)|<\eta$ for all $\xi \in \mathbb{P}^{d-1}$ and

$$
\left\|\tilde{v}_{\delta}-v_{\delta, n}\right\|_{\infty}<\tilde{\varepsilon}
$$

From [12] it follows that $\eta>0$ and $\tilde{\varepsilon}>0$ can be made arbitrarily small using a suitable grid; furthermore $\tilde{v}_{\delta}$ is Hoelder continuous, i.e. it satisfies

$$
\left|\tilde{v}_{\delta}\left(\xi_{1}\right)-\tilde{v}_{\delta}\left(\xi_{2}\right)\right| \leq K d\left(\xi_{1}, \xi_{2}\right)^{\gamma}
$$

where $\gamma \in(0.1]$ is an appropriate constant. Throughout this section $K>0$ will denote several appropriate constants. Note that also $v_{\delta, n}$ is Hoelder continuous, see [10].

We will now use $\tilde{v}_{\delta}$ in order to construct an approximately optimal feedback.

Definition 8 Define $\tilde{F}: \mathbb{P}^{d-1} \rightarrow U_{n}$ as follows. For any point $\xi \in \mathbb{P}^{d-1}$ choose a value 


$$
q(\xi, u)+\mathrm{e}^{-\delta} \tilde{v}_{\delta}(\varphi(1 ; \xi, u))
$$

becomes minimal and let $\tilde{F}(\xi):=u$.

The previous definition yields an approximately optimal feedback law.

Proposition 9 Let $\tilde{F}: \mathbb{P}^{d-1} \rightarrow U_{n}$ be a feedback law obtained from Definition 8 . Then the following inequality holds

$$
\begin{aligned}
\left\|\sum_{s=0}^{\infty} \mathrm{e}^{-\delta s} q\left(\varphi\left(s ; \xi_{0}, \tilde{F}\right), \tilde{F}\left(\varphi\left(s ; \xi_{0}, \tilde{F}\right)\right)\right)-v_{\delta, n}\left(\xi_{0}\right)\right\| & \leq \sum_{s=0}^{\infty} \mathrm{e}^{-\delta s} \eta\left(\varphi\left(s ; \xi_{0}, \tilde{F}\right)\right)+\tilde{\varepsilon} \\
& \leq \frac{\eta}{1-\mathrm{e}^{-\delta}}+\tilde{\varepsilon}
\end{aligned}
$$

for all $\xi_{0} \in \mathbb{P}^{d-1}$.

PROOF. From Definition 8 and the first assumption on $\tilde{v}_{\delta}$ it follows, that

$$
\tilde{v}_{\delta}(\xi)=q(\xi, \tilde{F}(\xi))+\mathrm{e}^{-\delta} \tilde{v}_{\delta}(\varphi(1 ; \xi, \tilde{F}))+\eta(\xi)
$$

for all $\xi \in \mathbb{P}^{d-1}$. Proceeding inductively with $\tilde{v}_{\delta}(\varphi(1 ; \xi, \tilde{F}))$ and using the second assumption on $\tilde{v}_{\delta}$ yields the first inequality. The second follows from $\sum_{s=0}^{\infty} \mathrm{e}^{-\delta s}=\frac{1}{1-\mathrm{e}^{-\delta}}$.

The feedback as constructed in Definition 8 does not possess any regularity properties, in particular it will in general be discontinuous. However, it is possible to approximate this feedback by a piecewise constant function which still yields approximately optimal trajectories.

Proposition 10 Let $\left\{V_{j} \mid j=1, \ldots, J\right\}$ be a family of disjoint sets with $\bigcup_{j=1}^{J} V_{j}=\mathbb{P}^{d-1}$ and $\sup \left\{d(\xi, \zeta) \mid \xi, \zeta \in V_{j}\right\} \leq \alpha$ for all $j=1, \ldots, J$. Define a feedback law $\tilde{F}_{\alpha}$ by

$$
\left.\tilde{F}_{\alpha}\right|_{V_{j}} \equiv \tilde{F}\left(\xi_{j}\right)
$$

for arbitrary (but fixed) points $\xi_{j} \in V_{j}$ and all $j=1, \ldots, J$ and $\tilde{F}$ from Definition 8.

Then for any $\varepsilon>0$ there exists a constant $\alpha>0$ such that for any approximation $\tilde{v}_{\delta}$ of $v_{\delta, n}$ with sufficiently small $\eta>0$ and $\tilde{\varepsilon}>0$ from (12) and (13) the following inequality holds for all $\xi \in \mathbb{P}^{d-1}$

$$
\left|\sum_{s=0}^{\infty} \mathrm{e}^{-\delta s} q\left(\varphi\left(s ; \xi, \tilde{F}_{\alpha}\right), \tilde{F}_{\alpha}\left(\varphi\left(s ; \xi, \tilde{F}_{\alpha}\right)\right)\right)-v_{\delta, n}(\xi)\right|<\varepsilon .
$$




$$
\tilde{v}_{\delta}\left(\xi_{j}\right)=q\left(\xi_{j}, \tilde{F}_{\alpha}\left(\xi_{j}\right)\right)+\mathrm{e}^{-\delta} \tilde{v}_{\delta}\left(\varphi\left(1 ; \xi_{j}, \tilde{F}_{\alpha}\right)\right)+\eta\left(\xi_{j}\right)
$$

Hence because of the Hoelder continuity of $\tilde{v}_{\delta}$ it holds for arbitrary $\xi \in V_{j}$

$$
\begin{aligned}
\tilde{v}_{\delta}(\xi) & =\tilde{v}_{\delta}\left(\xi_{j}\right)+K_{1}(\xi) \alpha^{\gamma} \\
& =q\left(\xi_{j}, \tilde{F}_{\alpha}\left(\xi_{j}\right)\right)+\mathrm{e}^{-\delta} \tilde{v}_{\delta}\left(\varphi\left(1 ; \xi_{j}, \tilde{F}_{\alpha}\right)\right)+\eta\left(\xi_{j}\right)+K_{1}(\xi) \alpha^{\gamma} \\
& =q\left(\xi, \tilde{F}_{\alpha}(\xi)\right)+\mathrm{e}^{-\delta} \tilde{v}_{\delta}\left(\varphi\left(1 ; \xi, \tilde{F}_{\alpha}\right)\right)+\eta\left(\xi_{j}\right)+K_{2}(\xi) \alpha^{\gamma}
\end{aligned}
$$

where $K_{2}(\xi)<K$ is a uniformly bounded function. Now the assertion follows by induction and the assumptions on $\tilde{v}_{\delta}$.

Note that the previous proposition can in particular be used for the construction of piecewise constant feedbacks by imposing further regularity conditions on the $V_{j}$. On way to obtain such sets is by using a partition (e.g. some triangulation) of the $d$ dimensional unit sphere and then by identifying $\mathbb{P}^{d-1}$ with one hemisphere. Observe that the construction of the piecewise constant map can also be based on the feedback $F_{\delta, n}$ from Definition 6 . The following theorem now states the main existence result for piecewise constant feedbacks.

Theorem 11 Consider system (1) and assume its associated projection (6) is forward accessible. Assume furthermore that inf $\Sigma_{F l}(C)<0$. Then there exists a piecewise constant feedback law $\tilde{F}_{\alpha}: \mathbb{P}^{d-1} \rightarrow U_{n}$ such that $J_{\delta}\left(\xi_{0}, \tilde{F}_{\alpha}\right)<0$ for all $\xi_{0} \in \mathbb{P}^{d-1}$, and the map $F: \mathbb{R}^{d} \rightarrow U_{n}$ given by $F(0)=u_{0}$ for $u_{0} \in U_{n}$ arbitrary and

$$
F(x)=\tilde{F}_{\alpha}(\xi) \quad \text { iff } \quad \mathbb{P} x=\xi
$$

defines an exponentially stabilizing piecewise constant feedback.

PROOF. From inf $\Sigma_{F l}(C)<0$, Theorem 7 and Proposition 10 the existence of $\tilde{F}_{\alpha}$ with the proposed properties follows. Hence we obtain

$$
\sum_{s=0}^{\infty} \mathrm{e}^{-\delta s} q\left(\varphi\left(s+t ; \xi, \tilde{F}_{\alpha}\right), \tilde{F}_{\alpha}\left(\varphi\left(s+t ; \xi, \tilde{F}_{\alpha}\right)\right)\right)<c
$$

for some value $c<0$ and all $t \in \mathbb{N}$. Thus the assumptions of [23, Proposition 3.8] are satisfied and we obtain $\lambda\left(x_{0}, F_{\alpha}\right)<c$ for all $x_{0} \in \mathbb{R}^{d} \backslash\{0\}$. As in the proof of Theorem 7 this implies exponential stability of the closed loop system on $\mathbb{R}^{d}$. 
For semi-linear systems whose projection satisfies a controllability assumption we have shown that open loop asymptotic null controllability and exponential feedback stabilizability via piecewise continuous maps is equivalent. For these feedbacks no explicit formula has been obtained and we also do not expect that a simple representation exists. Rather a numerical procedure for their construction has been presented.

Finally let us briefly comment on the robustness of the proposed stabilization scheme. One reason why feedback laws are preferred to open loop controls is that one expects some robustness of the stabilization against small errors or perturbations.

Unfortunately, $F_{\delta, n}$ as well as $\tilde{F}$ and $\tilde{F}_{\alpha}$ are in general discontinuous, hence continuous dependence on the initial value will not hold for the closed loop system and thus not yield the desired robustness result. Nevertheless it is possible to show that the feedback controlled trajectories are robust in the sense that they remain approximately optimal if the system is subject to small perturbations using discrete-time versions of Proposition 5.2 in [14].

Acknowledgments: The authors want to thank Luiz San Martin for suggesting Example 4.

\section{References}

[1] F. Albertini and E. Sontag, Discrete-time transitivity and accessibility: analytic systems, SIAM J. Control Optim., 31 (1993) 1599-1622.

[2] J.N. Tsitsiklis and V. Blondel, The Lyapunov exponent and joint spectral radius of pairs of matrices are hard - when not impossible - to compute and to approximate, Math. Contr., Sign., and Syst., 10 (1997) 31-40.

[3] C. Simoes, H. Nijmeijer and J. Tsinias, Nonsmooth stabilizability and feedback linearization of discrete-time nonlinear systems, Int. J. Robust Nonlinear Control, 6 No.3 (1996) 171-188.

[4] S. Celikovský, On the stabilization of the homogeneous bilinear systems, Syst. Cont. Lett., 21 (1993) 503-510.

[5] L.-K. Chen, X. Yang, and R.R. Mohler, Stability analysis of bilinear systems, IEEE Trans. Autom. Control 36 (1991) 1310-1315.

[6] F.H. Clarke, Yu.S. Ledyaev, E.D. Sontag and A.I. Subbotin, Asymptotic controllability implies feedback stabilization, IEEE Trans. Autom. Control 42 (1997) 1394-1407.

[7] F. Colonius and W. Kliemann, Linear control semigroups acting on projective space, $J$. Dynamics Diff. Equations 5 (1993) 495-528.

[8] F. Colonius and W. Kliemann, The Lyapunov spectrum of families of time varying matrices, Trans. Amer. Math. Soc., 348 (1996) 4389-4408.

[9] F. Colonius, W. Kliemann, and S. Krull, Stability and stabilization of linear uncertain systems - a Lyapunov exponents approach. Report 372, Schwerpunktprogramm der Deutschen 
Augsburg, 1992.

[10] L. Grüne, Discrete feedback stabilization of semilinear control systems, ESAIM: Control, Optimisation and Calculus of Variations 1 (1996) 207-224.

[11] L. Grüne, Numerical stabilization of bilinear control systems, SIAM J. Contr. BS Opt. 34 (1996) $2024-2050$.

[12] L. Grüne, An adaptive grid scheme for the discrete Hamilton-Jacobi-Bellman equation, Numer. Math. 75 (1997) 319-337.

[13] L. Grüne, Asymptotic Controllability and Exponential Stabilization of Nonlinear Control Systems at Singular Points, SIAM J. Contr. \& Opt. 36 (1998) 1585-1603.

[14] L. Grüne, Input-to-state stability of exponentially stabilized semilinear control systems with inhomogeneous perturbations. Preprint No. 98/23, Dipartimento di Matematica "Guido Castelnouvo", Universit di Roma "La Sapienza", 1998. submitted.

[15] A.Y. Khapalov and R.R. Mohler, Asymptotic stabilization of the bilinear time-invariant system via piecewise-constant feedback, Syst. Cont. Lett. 33 (1998) 47-54.

[16] W. Lin, Further results on global stabilization of discrete nonlinear systems, Syst. Cont. Lett. 29 (1996) 51-59.

[17] W. Lin and C.I. Byrnes, Design of discrete-time nonlinear control systems via smooth feedback, IEEE Trans. Autom. Control 39 (1994) 2340-2346.

[18] W. Lin and C.I. Byrnes, KYP lemma, state feedback and dynamic output feedback in discretetime bilinear systems, Syst. Cont. Lett. 23 (1994) 127-136.

[19] E.P. Ryan and N.J. Buckingham, On asymptotically stabilizing feedback control of bilinear systems, IEEE Trans. Autom. Control, 28 (1983) 863-864.

[20] E. D. Sontag and F. R. Wirth, Remarks on universal nonsingular controls for discrete-time systems, Syst. Cont. Lett., 33 (1998) 81-88.

[21] Y. Stepanenko and X. Yang, Stabilizing controllers for discrete bilinear systems, Int. J. Robust 83 Nonlinear Control, 6 (1996) 855-867.

[22] H. Wang, Feedback Stabilization of Bilinear Control Systems, SIAM J. Contr. \& Opt. 36 (1998) $1669-1684$.

[23] F. Wirth, Asymptotics of value functions of discrete-time discounted optimal control, Report 411, Institut für Dynamische Systeme, Universität Bremen, 1997. submitted.

[24] F. Wirth, Dynamics of time-varying discrete-time linear systems: Spectral theory and the projected system, SIAM J. Contr. \& Opt. 36 (1998) 447-487.

[25] X. Yang, L.-K. Chen, and R.M. Burton, Stability of discrete bilinear systems with output feedback, Int. J. of Control, 50 (1989) 2085-2092. 